International Journal of Education (IJE) Vol.4, No.4, December 2016

\title{
EMBODYING THE EDUCATIONAL INSTITUTION
}

\author{
Matthew Montebello \\ Department of Intelligent Computer Systems, \\ Faculty of ICT, University of Malta, Malta
}

\begin{abstract}
The way adults pursue their education through life is changing as the technology around us relentlessly continues to enhance our quality of life and further enhances every aspect of the different tasks we set out to perform. This exploratory paper looks into how every adult can embody a comprehensive set of academic services, platforms and systems to assist every individual in the educational goals that one sets. A combination of three distinct technologies are presented together with how they not only come together but complement each other around a person in what is usually referred to as a personal area network. The network in this case incorporates an intelligent personal learning environment providing personalised content, intelligent wearables closer to the user to provide additional contextual customisation, and a surrounding ambient intelligent environment to close a trio of technologies around every individual. Each of the three research domains will be presented to uncover how each contributes to the personal network that embodies what one usually expects from an educational institution. Three distinct prototype systems have been developed, tested and deployed within a functional system that will be presented in this paper.
\end{abstract}

\section{KEYWORDS}

Higher Education, Personal Learning Environment, Intelligent Wearable's, Ambient Intelligence, Personal Area Network.

\section{INTRODUCTION}

Further and higher education institutions are struggling to keep afloat as financial and operational difficulties have and continue to shift within a multi-facetted domain that is dealing with issues related to competition, costs, human resources, accreditation, assessment, and quality assurance to mention a few. Even the way and the quality of teaching and learning has shifted due to these issues as educational institutions and academics had to adjust together with their epistemological standing and pedagogical philosophy adopted. The high level group on the modernisation of higher education[1] pointed out that the "pedagogical models designed for small institutions catering to an elite few are having to adapt, often under pressure, to the much morevaried needs of the many, to greater diversification and specialisation within higher education, to new technology-enabled forms of delivery of education programmes, as well as to massive changes in science, technology, medicine, social and political sciences, the world of work, and to the onward march of democracy and human and civil rights discourses." (pp.12) The impact of technology

DOI : $10.5121 /$ ije. 2016.4402 
mentioned above is instrumental to a number of efforts to adapt to the changing times. Distance education and eventually e-learning are different forms of delivery triggered by massive strides in technology but which still carry their own challenges and limitations. The learner on the other hand has to adapt to whatever the educational institution has to offer, which does not always mean or result in an optimal outcome. As a matter of fact the demographics together with the retention and students' success rates tell a story of their own. Such matters within a competitive and globalised higher education market have serious repercussions and damaging to an educational institution [2]. The employment of technology within e-learning in an attempt to alleviate these issues is a typical example of how some higher education institutions fail to focus on the learner and instead assume that the technology will suffice. Macfadyen and Dawson [3] point out that in reality those institutions that dominate their planning process by technical concerns rather than what the learners need and focus on, "fail to develop a clear vision for learning technologies" (pp. 161). Additionally, Beverly Park Woolf[4] specifically underlines this issue when she attributes the shortcomings of instructional software to its inability to truly respond to student needs and inflexibility to emulate teaching. In this paper a learner-centric methodology will be presented as it embodies the basic elements of an academic institution. The integrated e-learning platform attempts to provide all the required education needs of a student with an essential personalisation element that tailors the content, delivery and feedback that a learner expects from a one-to-one interaction with an educator.

The rest of the paper is organised as follows. The next section will cover the background necessary to better understand the concepts that make up the integrated platform. This involves the use of personal learning environments, the input from wearable and portable technologies, the contribution of ambient intelligent surroundings, and the integrating medium of a personal area network. The way these technologies and techniques converge together will be presented in Section 3 where three distinct and autonomous prototypes are described in detail. In the next section the test models are presented together with the documentation of the full set of results. The future work and conclusions bring this paper to a close as they are presented in Section 5 and Section 6 respectively.

\section{BACKGROUND}

The techniques and technologies presented in this section will help set the scene for their employment in the next section. These distinct areas are research domain in their own right and yet when combined together they promise to deliver more than they individually can as each adds value and complement each other. The first to be presented is the Personal Learning Environment or PLE that is not something new or innovative but which still plays an important role when academic personalisation needs to be achieved. Secondly, every intelligent system requires input from the user or from sensors like wearable and mobile devices as they are located in close vicinity and part of the same user. As a matter of fact the second closest distance to the user is his or her environment, and this can also serve as a sensory input but also as an output medium, and this will be the third to be presented. Finally, the personal area network or PAN is simply a concept and a technology that allows the previous three presented areas to coexist, interact, and provide combined added value to the user. 


\subsection{Personal Learning Environment (PLE)}

The inception of combining a number of techniques originated from the educational relevance and use of a Personal Learning Environment (PLE). The reason for adopting the use of personal learning environments is entirely due to their capability of personalising the learning process and creating an environment within which the student can be truly immersed. A Personal Learning Environment, which, in contrast to a course-centric learning management system, such an environment is student-centric, and predominantly constitutes an academically tailored environment [5]. A personal learning environment, as shown in Figure 1, has two dimensions to it, namely, a Personal Learning Network (PLN) and a Personal Learning Portfolio (PLP). Personal learning networks are traditionally considered to encompass the online communities learners are registered with, and with whom they engage with to contribute and exchange information [6]. On the other hand, personal learning portfolios are considered to be a collection of works that reflect a student's academic efforts, progress and accomplishments [7]. These two dimensions, the PLN and the PLP, together with the outcome of their combination, the personal learning environment, overlap the other approaches that will be covered in the next sections.

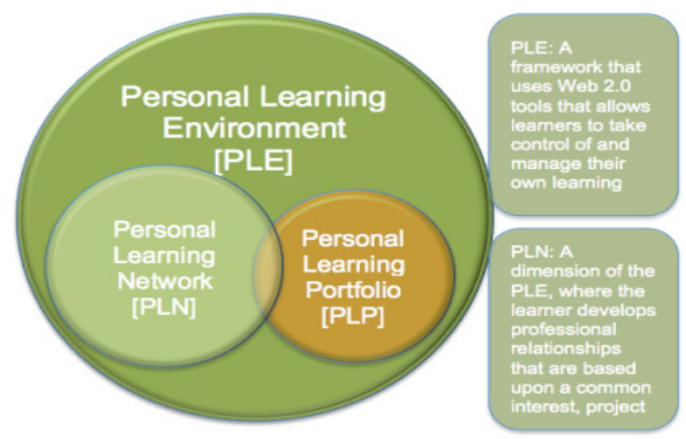

Figure 1 - A visual representation of a PLE[8]

\subsubsection{Personal Learning Network (PLN)}

Leone [6] emphasises the importance of a learner support system made up of social connections and online resources. This learning network is unique to individual learners as it evolves over time and through continuous interaction that will eventually contribute to the personal and professional development and knowledge. Personal Learning Networks (PLNs) are firmly set within the connectivism learning theory and their ultimate goal is to empower learners and educators by building a personal community of peers and knowledge providers online in a way to share, collaborate and source information, ideas and knowledge. The potential of having a massive online knowledge base at one's fingertips is intense and overwhelmingly powerful that is sometimes overlooked and not taken advantage of. To build such a network a person needs the adequate tools, social networking tools, to be able to connect and interact with other web users who likewise are developing their own personal network. Every individual can decide on the way to go about extending one's network while at the same time defining the way to learn, what to learn, and at which pace. Such networks automatically promote collaboration and sharing thereby fostering a communal sense of belonging and non-isolation. Developing a private learning network is not a simple task or a decision following an impulse to do so, but a mind-set and a way of life. It is a conscious choice of continuous learning, a dedication to search, collect and curate interesting information, and a passion to create, distribute, share and collaborate with other likeminded people while employing the Internet as a communication medium. Typically a minimal 
set of tools and activities required for a personal learning network require one or more social networking accounts to link up and communicate with other social networkers who have similar interests and needs; follow, contribute and distribute content discovered or generated over a blog, a wiki or any other social bookmarking online tool; join and participate in discussion groups, fora and other social gatherings to acquire new information while at the same time sharing personal knowledge with others. Much of these online tools have been made available and are possible through the advent of Web 2.0 technologies [5] that characteristically present dynamic rather than static websites displaying user-generated content.

The personal learning network element will be put into practice through the use of crowdsourcing and the generic use of Web 2.0 technologies. Crowdsourcing is a simple practice of making use of the collective knowledge of potential contributors to solve a common problem or to complete a specific task. Such a practice is commonly applied and functionally proven within industry [9] as overtly proven by the Open Source success within the ICT arena. There have been numerous other situations where huge numbers of contributors have factored into the final overall success of what they collectively had a vested interest in. Some familiar examples include Wikipedia, Linux, Yahoo! Answers, and Mechanical Turk [10]. The concept of harnessing the power of the masses has also been applied within a variety of domains. Bernstein, et al.,[11] report how crowdsourcing was applied to a word processing application, while in another related project [12] investigates how interfaces can be controlled by crowd input. In another project Christian, Lintott, Smith, Fortson, \&Bamford, (2012) apply crowdsourcing to the domain of astronomy. Other examples can be found in journalism [13], art [14], medicine [15], government [16], and finance [17]. Typically in industry, such a practice is employed to disseminate a problem a company has encountered to all their collaborators and partners. These in turn send back potential solutions and improvements that the same company collates and adopts to upgrade or enrich their product. Literat[18] summed up the essence of crowdsourcing when she identified four cornerstones of crowdsourcing, namely, connecting, communicating, collaborating, and learning collectively. Within the context of this paper crowdsourcing refers to publically available resources, as well as, content and contributions made available online. In particular, this research evaluates the utilisation of crowdsourcing within the higher education domain, as one of the practices employed to add value to e-learning. My interpretation of crowdsourcing in this respect is simply the application and practical use of data provided over social networks to the domain of education. The concept of employing crowdsourcing to higher education is a relatively novel research area, but not a new one. It can be evidenced in a framework presented by [19] whereby they aimed to evaluate active learning in conjunction with crowdsourcing. The improvement in classification performance was compared to state-of-the-art methodologies and concluded that crowdsourcing is a promising practice when applied to education, but noted that it could be fallible in accomplishing specific tasks. They recommend that a thorough crowd evaluation is required before applying the collective intelligence to work on a specific issue. Weld, et al., [20] offer a direct association between online education and crowdsourcing, as they argue that such a mechanism will likely be essential in order for e-learning to reach its full potential. The use of social networks and the fact that crowdsourcing is considered to be leveraging available online platforms is only part of its advantages. Another advantage that needs to be kept in mind is the fact that it is a low cost and scalable method to source and mediate such rich and diverse information sources [9]. The diversity of socially contributed opinions can actually diminish bias especially in collective decision making within small teams as reported by Bonabeau[21]. This is particularly true when one considers the financial repercussions of adopting a virtual crowdsourcing panel of experts in contrast to a team of real professionals. Crowdsourcing comes with its own issues and challenges especially due to the heterogeneous and unstructured nature of 
International Journal of Education (IJE) Vol.4, No.4, December 2016

the content itself. Web-based content especially that over social networks is predominately not reviewed or humanly moderated, thereby creating a massive challenge to parse and compose information that is valid and adequate for academic consumption. Some solutions lend themselves very well already as they are easily separable into well-defined manageable sources. Tools like ConsiderIt ${ }^{1}$, SuggestBot[22], and Soylent ${ }^{2}$, further assist to facilitate and alleviate this classic yet fundamental crowdsourcing concern.

Another issue worth keeping in mind when considering social networks in conjunction with education is the element of student engagement in relation to the connectivism learning theory that will be discussed later in the chapter. Studies have clearly showed that there exists a direct correlation between social networking and engagement. [23] have statistically confirmed, through analyses of Twitter communications, that "students and faculty were both highly engaged in the learning process in ways that transcended traditional classroom activities" (p.1). Their study provided "experimental evidence that Twitter can be used as an educational tool to help engage students and to mobilize faculty into a more active and participatory role" (p.1). Similarly, Rutherford [24] has shown that there is a positive correlation between student use of social media and the quality of their educational experience. The study gave positive insights into the impact the use of social media can have on the level of pre-service student engagement. Other studies[23] have also shown that leveraging social networks during the educational process enhances student engagement.

They provide the required connections between users thereby facilitating communication, collaboration, and collective learning at the same time. These are the reasons that led to the adoption of social networks within the context of crowdsourcing as part of the personal learning network component. The combination of education and crowdsourcing research is being argued to be a natural blend that potentially offers fruitful outcomes. At classroom level, an educator is more than willing, excited, and eager, to share knowledge and convey information. The same educator employs the most appropriate medium to optimise the communication transaction, while ensuring the most efficient educational process. This is not always the case unfortunately, but it simply demonstrates that even though the educator might have the best of intentions to teach, and the student is willing to learn, communication problems are possible. Creating the most favourable environment to reduce the possibility of communication break downs is the least that could be done. In the case of crowdsourcing, a favourable environment could be supported by providing adequate tools and applications to encourage and engage providers and consumers. The same consumers, in a recursive manner, become contributors and providers, thereby creating a cycle within a collaborative healthy eco-system that implicitly spawn educational processes. The research project assumes an idealistic scenario whereby educators enthusiastically contribute and facilitate the educational process through their collaboration, passion and vocation. Crowdsourcing provides the opportunity to harness the power of the crowd and deal with the vast amounts of potentially useful data provided by massive numbers of users, contributors, academics and experts. The challenge here that this research study is attempting to address is to process the massive amounts of data available, which is otherwise humanly laborious to do, and use it fruitfully. The outcome will be a freely available resource that provides content as part of the learner's personal learning network.

\footnotetext{
${ }^{1}$ http://consider.it/

${ }^{2}$ http://projects.csail.mit.edu/soylent/
} 
International Journal of Education (IJE) Vol.4, No.4, December 2016

\subsubsection{Personal Learning Portfolio (PLP)}

The Personal Learning Portfolio (PLP) is the second component within the personal learning environment that constitutes the second approach adopted during this research. [7]talk about a collection of a student's work that characterises her/his academic record. The authors argue that such portfolios have evolved over the years from traditional physical learning portfolios to the elearning domain in order to personalise learning. [25]describe such portfolios as valuable learning tools that go beyond the simplicity of an electronic collection of student artefacts. As a matter of fact the authors identify six categories of personal learning portfolios amongst which is a learner profiling functionality that employs the portfolio to plan educational content in line with the unique characteristics of the student. To this extent [26] suggest that, based on latest research, personal learning portfolios are "practical tools for supporting self-directed and reflective learning" (p.231). As a matter of fact in the next section I will be coupling this second technique with the self-determination learning theory in an effort to address the motivation issues learners reported in respect to e-learning systems. This is confirmed by other studies [27][7] that highlight the escalation of student enthusiasm to further participate and take initiative in their learning process. In this respect [27] attribute cognitive improvement, a rise in individualised learning, and overall improvement in the e-learning medium. Furthermore, [26] state that portfolios also support collaborative learning whereby learners share their work and resources for educational purposes. This is perfectly inline with the personal learning network concept discussed at the beginning of this section. [28]also highlights this coupling as he concludes that through the use of personal learning portfolios within a personal learning environment learners are able to capture and manage their knowledge status. Furthermore, the author remarks that the educational process can improve if the same learners socially engage and strike connections within their peer community to discuss, contribute and share content. The close correlation between learner profiling and personal learning portfolio is also acknowledged in the research reported by [29] who confirm that personal learning portfolios are ideal sources of information to initialise learner models that are eventually employed to create adaptive educational material. They highlight the benefits of learner profiling and how such an approach is strategic to reflective and personalised learning. A learner profile contains specific and essential information related to the academic persona of a unique student. Such profiles represent a direct mapping to the distinctive characteristics of individual students as they differ in their academic background, interests, preferences, and learning goals. The student could be initially asked to explicitly declare the specific qualities, descriptions or characteristics that can be employed to develop the profile. On the other hand, numerous simple learner profile generators automatically develop the required profile that can be used to personalise the service being rendered [30]. A well-known and basic issue with automatic profile generators is the inability to produce a profile at the very beginning of the process when no previous information about the learner is available. This problem commonly referred to as the 'cold start' effect [31] can be easily and quickly addressed by adopting the explicit collection of learner interests and needs at the beginning of the process, and eventually employ automatic profile generation from then onwards. The initial explicit method generates enough information and momentum for the automatic method to seamlessly take over the process and effectively generates a learner profile that can be productively used to personalise the content. The content that is presented is highly dependable on the application area under consideration together with the reasons for doing so. In the case of online information systems like newspapers the generated profiles would characteristically contain the reading habits and patterns together with topical items the readers are interested in, while ensuring not to include others that they dislike. Another domain dependent example is a personal scheduling system where the profile generated ensures to take into account not just the date, time, venue and 
participants, but also personal priority issues together with re/scheduling habits and patterns. Within the academic domain the profile generated encapsulates as much as possible the comprehensive learner characteristics that deal with knowledge, interests, and educational needs. In this respect a learner profile is considered a collection of inferences about information concerning a student that one is not able to observe [32]. The main use of the learner profile is to adapt and personalise the learning process as well as the content and the delivery of the educational material. An automated learner profile can be generated using Computer Science techniques that go beyond the scope of this paper but for completeness sake the most commonly employed profiling techniques will be highlighted shortly. Adding value to services and personalising the information presented to the user is a very well researched domain within the ICT arena [33]. Numerous methods are available to generate a specific user profile that will eventually be employed to tailor the information intended for user consumption [34]. In this context the students' individual profile can be employed to identify and adapt the educational content for their focused use. Such practices have been successfully employed to generate user profiles and personalise the service provided to the same users.[35]employ user profiles to personalise information access to control the information overload problem experienced by web users. Similarly, the same concept was applied to healthcare consumers [36], e-commerce applications [37], and mobile guides [38]. Within the educational domain there have been several research projects and initiatives that employed personalisation to deliver educational content [39]. The use of online resources and web services have also been investigated by researchers like[40] to enrich e-learning in terms of personalised learning systems, where the system itself was able to trace learner needs and track progress. This would fit very well with the proposed research whereby large amounts of users could potentially possess individual and unique academic profiles. As a matter of fact [41] document how research in e-learning started taking form in a way that also involved the tailoring of tools, terminals, and communications, to the needs of the individual people as captured through their profiles, needs and experiences. In this context, a number of authors started describing the use of developing the right technologies to be able to provide a more 'effective' e-learning service [42]. Most often this type of research was rather detached from the complexity of the human learning involved, and this is why such systems were morphed into personalised learning networks more than simple systems [43]. What becomes obvious from such setups is the convergence to an idealistic learning scenario. A textbook model would be a one-to-one student-teacher set-up whereby the content provided is perfectly tailored to the student's needs and interests. Such personalisation is what this research study is aiming to investigate within a wider context and with the help of additional online tools. Three of the most widely employed and artificial intelligent techniques employed to generate a user profile will be briefly presented and brought into context.

Association rules are simple directives that imply specific associations or correlated relationships between groups of items within a specific domain. Such rules are generally employed to discover patterns from data collected. Given a set of facts about the user's academic achievements, and an associated list of tasks and topics, the rules formalise the connotations between them. For the sake of simplicity, a classic example involves shopping trends of consumers. It is common knowledge that whoever purchases wine and cheese, the chances of purchasing grapes as well is about $75 \%$. Such a rule, with an associated numeric value, can work out what future consumers will likely to go for after purchasing cheese and grapes. Association rules have been applied to different domains like large databases, eCommerce, and education. If such rules were to be employed, then some previous knowledge of students' trends and interest will be required to create the required association rules. 
International Journal of Education (IJE) Vol.4, No.4, December 2016

Another artificially intelligent technique used to generate a user profile is called case-based reasoning that makes use of previous cases that are similar to the current problem at hand and applies or adopts the solution to the situation. So, given a student who has a problem with understanding a particular academic topic, the case-based reasoner retrieves relevant cases that match such a request and adapts solutions that were effective to solve the similar problem. The difficult part for the classification task arises when the system is required to identify a target class for a case that has no classification. In such instances the solution to this dilemma is simply fitting the class that is most similar. Case-based reasoning has been employed as a learner profile generator in various customisation scenarios like web information searching, topical filtering of data, and document clustering.

The final computer science technique that is commonly used to create a user profile automatically is through the use of Bayesian networks. A network is simply a set of points interconnected to each other with links or lines. This system makes use of lines to link items of interest that are related to each other. This means that there is no relationship between disconnected topics of interest to a student. On the other hand a subject that is connected to another topical matter, that in turn is connected to a third theme, automatically infers a transitional association, thereby proposing the third item as a potential topic of interest to the student. [44]make extensive use of such networks to capture students behaviour within an e-learning system and model their learning styles. Other uses include web browsing personalisation, intelligent help systems, and expert systems.

What emerges from the above brief descriptions is that any of these basic techniques can be employed to generate a student profile and personalise the content that is presented to the same student. This does not mean that they all perform equally but that they do so differently. The ultimate goal, as far as this paper is concerned, is that an artificial intelligent piece of software is applied as part of the personal learning portfolio component, which together with the personal learning network make up the e-learning personal learning environment under investigation. Whereas the functionality of the personal learning network component is achieved through the use of crowdsourced social networks, the personal learning portfolio component is implemented through a simple process of user profile generation that is sourced through the combination of explicit interest declaration and the eventual interactions with the environment. The learners' feedback is used to refine the generated profile to better personalise the educational content that follows.

\subsubsection{PLE and Personalisation}

The combination of a personal learning network (PLN) and a portfolio (PLP) help in establishing an environment that is not only personal, but even more effective due to its customised and tailored content that fits even closer to the user's needs and interests. Siemens [44] explored aspects of personalised learning with a focus on how to connect all the information provided online in a way which makes sense in context; using networks to help amalgamate all the information acquired in a meaningful way. [45]report how these technologies connect knowledge workers to their online personal networks for information exchange, informal learning, and social support, thereby supporting the notion of a personal learning network that has value-added advantages due to the use of social media. The personal learning environment brings together the two components (PLN and PLP) in a conceptual way within an integrated e-learning user interface. [46] have teamed up in an attempt to integrate personalisation in the online courses they offer. This partnership that started in 2013 has launched a full scale initiative earlier this year by 
offering four hundred thousand first year university students the possibility to make use of personalised educational services. Tailored feedback and customised academic advice was delivered based on information that was extracted from the same students' success and failure patterns while going through the educational material. Another interesting partnership was struck between University of Edinburgh and CogBooks who developed an online tool that personalises the students' graphic user interface as they progress through the different course activities [47]. The academics in return have used this same information to fine-tune their material and teaching in general. Two other similar partnership between CogBooks and Arizona State University and University of Colorado Boulder have also been using personalisation techniques to provide formative feedback to individual students based on analysis of learner-generated data [48]. CogBooks are encouraged by the result obtained and claim that they are successfully achieving their goal of 'educating everyone uniquely' [49]. Similar results were reported by the University of Wisconsin-Milwaukee [50] who developed a self-paced programme called U-Pace that incorporates personalised feedback, individualised progress reports, and motivational notifications to make students aware of their strengths and weaknesses. The outcome showed that students performed sixteen percent higher on assessments over those who did not follow the UPace programme. This trend is also evidenced in courses being offered by MITx[51], the MIT wing that gives away free online courses, with the premise that specific students, as a result of their declared needs and interests, might be presented with variations in the academic content presentation. Two other related initiatives worth mentioning are those established by the Bill \& Melinda Gates Foundation, and the IMS Global Learning Consortium. The Gates foundation set up a grant program called ALMAP (Adaptive Learning Market Acceleration Program) that promoted personalized learning research, while setting up also a ground-breaking learning program called Enlearn whose purpose was to assist and encourage the development of adaptive learning material that can enable a more personalized teaching and learning experience thereby transforming the entire classroom ecosystem into an adaptive environment suitable to the learning needs of each student. On the other hand the IMS initiative brought together a consortium of over three hundred universities, higher-education institutions and vendors in an effort to standardize and establish a shared vocabulary for recording students' academic data. The protocol of metrics, called Caliper[52], was intended to make it easier to describe a learner's profile across institutions and learning environments.

It is obvious and natural that a human educator is much more effective when a personalized methodology is employed. Within an e-learning environment such recognition is also being confirmed as institutions across the world agree that a single invariable and inflexible style, method or approach is not possible for all learners [53]. Lonn et al.,[54] define personalized learning in a way that higher education institutions can take technological advantage through "the measurement, collection, analysis and report of data about learners and their contexts, for purposes of understanding and optimizing learning and the environments in which it occurs" ( $p$. 4). The on-going research in this area is on the rise as access to data tools and techniques are easier to use and highly accessible together with the availability of large sets of data that will assist in the customisation of the learning process.

\subsection{Wearable Technologies}

We are witnessing a shift to ubiquitous computing that has made it possible to have intelligent systems operate as effectively on mobile devices, and deployed in various scenarios without 
International Journal of Education (IJE) Vol.4, No.4, December 2016

compromising on performance while incorporating technologies such as recommendation systems. One scenario where such systems can be deployed effectively is in the higher education domain in the form of personalized e-learning.

The aim of this component is to make such wearable's act intelligently by having the device generate recommendations tailor made for the individual. Acting intelligently also involves the presentation of relevant information to the user at the time when this is actually required. Such tasks involve the implementation of user-profiling mechanisms in order to be able to understand the traits of the user and in turn generate recommendations that are as accurate as possible. Deployed in the aforementioned domain, such devices would ensure that any learner making use of an e-learning system gets the opportunity to take full advantage of the services being deployed without hindering the learning process with the cumbersome tasks of having to carry with him devices which are not very user friendly. Therefore, finding the right techniques with which to gather information, present it in structured formats, and more importantly, infer user traits from the data at hand is pivotal in the creation of such systems.

Research carried out focused primarily on personalization systems, mainly on how to construct efficient and effective user profiles. The ultimate goal of user-adaptive systems is to provide users with what they need without them asking for it explicitly.

\subsection{Personal Area Networks}

A Personal Area Network (PAN) is a normal computer network that is used for communication and transmission of data between different points or devices on the same network. The only difference from the traditional LAN (Local Area Network) or WAN (Wide Area Network) is that a PAN around a person and the devices on the network are the individual's personal devices. These may include mobile telephone as an input and output device, a mini camera as an input device, miniature display screen within glasses as an output device, storage medium around the waist or in a bag, wearable's as input devices, headphones as output devices, and others (Figure 2). Obviously a PAN is distinguishable from the other data networks due to its smaller size but also due to its scope, so in this case a PAN is expected to have a locus of less than ten metres enough for a person's devices and his or her surroundings to be able to connect and communicate. As a matter of fact it makes most sense for the PAN to be wireless thereby giving much greater freedom of movement to the individual. For this reason such a network is also sometimes referred to a Personal Operating Space (POS) as the interconnected devices centered around the user create a personalized workspace communicate usually through Bluetooth or Infrared (IR). The PAN also incorporate access to software that could potentially be running locally on the person's mobile phone device or could also be communicating to a larger network, like the WWW, through a WIFI connection through the same mobile phone. Advantages of a PAN includes its ability to focus on the user's devices only excluding all others that are not registered on the network, but also allows the possibility of connecting with other networks the user has control over like the home network, or the car, or the office. Issues of security need also be considered as personal information related to identity management, access control, reputation management, and financial matters require attention [55]. 


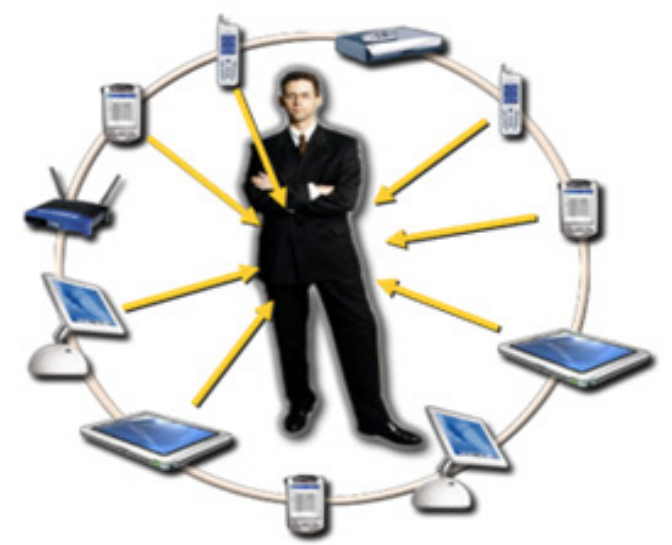

Figure 2 - Personal Area Network [55]

\section{CONVERGENCE OF TECHNOLOGIES}

The technologies highlighted in the previous section have been implemented within functional prototypes as a proof-of-concept to show that a personal educational network (PEN) is possible following best practices. The PLE developed has already been widely accepted in academia [56] and even though it has been through extensive testing, the intelligent PLE is now being employed in conjunction with other technologies as well as evolving to accommodate latest developments. The intelligent wearable's have also been developed as a proof-of-concept within another domain but have now been adapted to serve as input sensors to the PEN. Finally, the ambient intelligent system is the encompassing system that homes both prototypes and ensures that the delivery and the communication with the user is consistent and effective.

\subsection{Intelligent PLE}

The intelligent personal learning environment is based on the standard PLE as described in Section 2.1.1 with the use of a PLN and a PLP. It was specifically designed to address a number of e-learning concerns and founded on sound learning theories as we shall see, but its main objective is to:

1. Keep the learner motivated as much as possible throughout the learning process by ensuring that the specific learning process is captured in some way that represents patterns in the needs, interests and enthrallments of the same learner.

2. Integrate the learner within a comprehensive learning society whose members are sources of information as much as they are recipients. The connectivism learning theory focuses on a digital society where every learner is not isolated but forms part of a healthy network of academic nodes. The personal learning network that each learner possesses embodies this effort and will form part of the proposed environment.

3. Personalize the learning process through the adaptation of the learning material while ensuring that the tailored medium optimizes the delivery of this academic content.

This prototype is mainly founded on these three objectives and founded upon a learning theory of Connectivism. In this respect a learning theory forms part of the pedagogical model adopted 
together with the educational material employed, the methodology engaged, sequence, medium through which it's presented and finally the overall success or not. Tomei[57] defines pedagogy "as the art and science of teaching children" (p. 1), and describes how the evolution of learning theories has transformed the pedagogical model from a state of submissive or receptive child and teacher knows-it-all, to a learner-centered and academic facilitator. Pedagogy must not be an ad hoc concept that is left to chance or not given enough thought and planning, but requires sound theoretical foundations especially within the area of technology-enhanced education. McKenzie [58] points out that it's because of a pedagogical model was not followed that numerous academic institutions had a low return on their technological investments. McKenzie was reacting to a statement by the US secretary of education, Dr Roderick Paige, who side-lined the importance of pedagogy and imposed changes that were not grounded in any learning theories.

Based on these factors, this documented research study together with the methodologies adopted are structured around a predominant learning theory, Connectivism. Connectivism[59] is considered by numerous researchers (Downes, 2008; Kop \& Hill, 2008; Duke, Harper, \& Johnston, 2013) as the leading learning theory in the digital age as social networks and learners' online presence is considered influential on their academic work and personal lives [60]. The authors argue that according to George Siemens' theory "online social network contacts represent a potential and valuable source of information" (p.142). This source of information is not enough and definitely does not constitute a complete learning environment. As a matter of fact $\mathrm{Ng}$ [61], amongst others (Hung, 2014; Duke, Harper, \& Johnston, 2013), asserts that learning theories that support online learning like connectivism need also take into consideration those teaching contexts that are not in real time (asynchronous) as these situations have a major impact on the learning outcome. In this respect Mayes \& De Freitas[62] actually argue against the adoption or need of new learning theories to accommodate the digital age and assert that all that is required for effective learning is the knowledge of how the underlying processes and theoretical constructs enable learning, be it face-to-face or over e-learning. The point being made here is that a learning theory adequate for learning within the digital area and applied to this e-learning research is not enough or complete in isolation. This is especially true when a combination of methodologies is being proposed to enhance the effectiveness of e-learning. As a matter of fact $\mathrm{Ng}$ (2015) subscribes to this same notion when he states that "It is inevitable that the blending of more than one learning theory in the design of a sequence of pedagogically sound learning activities would be required" (p.93).

The connectivism learning theory has been associated with the use of social media in education, and coined as a "learning theory for the digital age" [63]. This theory puts into context the online reality of learners making use of social networks as it "dismisses the three dominant learning theories, behaviourism, cognitivism, and constructivism", according to Wheeler (2012). The educational process is envisaged external to the learner within a personal network of technologies, communities and social media. Closely related to this definition also lies the socialconstructivism theory that according to Vygotsky[64] learning occurs as a result of interactions between individuals influenced by the cultural and societal environment. Whereas this learning theory takes into consideration the role of others within the learning process as mediators to acquire novel information and knowledge, connectivism takes it a step further and highlights the importance of the networked information whereby the learner and the mediators contribute and receive in a mutual beneficial learning community. I particularly argue that the connectivism learning theory significantly contributes to this research project as it highlights the importance of learners identifying the source and the content itself of what interests them and what they need to learn. This places the responsibility directly on the learner who is required to bring together a 
cohesive set of personal learning tools within an environment that is socially networked and academically healthy within which learners can store their knowledge. Such an ideology subscribes to my own post-positivistic epistemological point of view whereby the contextual reality of an online experience determines and distinguishes our overall interaction and the amount and quality of what we intellectually extract. The medium employed is clearly an imperative factor in the facilitation of the learning process. The extent and capacity of the medium's influence is also dependent on the student at the receiving end of this interaction. A number of educational studies have been reported that directly refer to the learning theory of connectivism. Loureiro and Bettencourt [65] investigated how to enhance the educational process by focussing on optimising such process within higher education by integrating Web 2.0 tools and subscribing to connectivism. Robson [66] took a step further to investigate the next generation of online courses by scrutinizing the content and processes of initial generations of elearning courses. He draws the conclusion that e-learning content is experiencing a shift in its underlying pedagogical theories from cognitive, instructive, and behaviourist to social, constructivist and connectivist. Even Duke, Harper, \& Johnston, [67] argue that connectivism's diversity through different networks is ideal to assist learners in the new generation to learn.

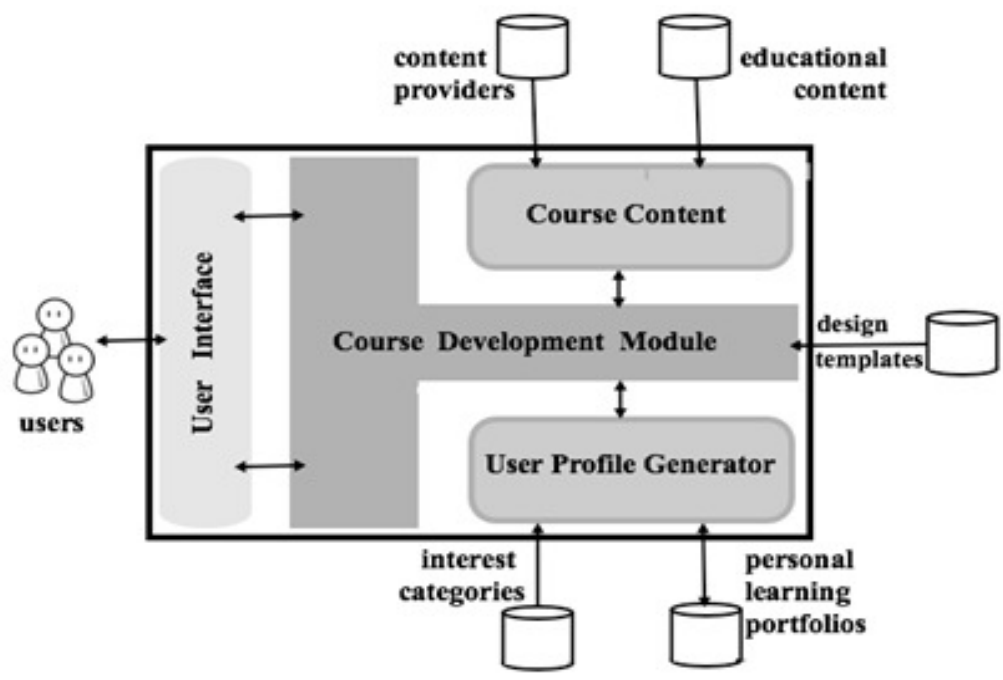

Figure 3 - The intelligent personal learning environment

They encourage educators to continually evaluate how connectivism in conjunction with other learning theories can be used in the online learning process. Furthermore, Hung [68], makes extensive use of ideas from this same learning theory to design new models in an effort to optimise the movement of connected knowledge, expanding learning spaces and structures, and employing open technology to connect people.

Based on this learning theory and implemented as an online course the intelligent personal environment (Figure 3) personalised the delivery of the educational material while at the same time employed the users' interests and interactions to personalise and further tailor the interface and the content alike. Machine learning techniques were employed to develop and evolve a learner profile while crowdsourcing and social networks, namely Facebook and Twitter, were employed to complement the content. 
International Journal of Education (IJE) Vol.4, No.4, December 2016

\subsection{Intelligent Sensors}

The intelligent sensors were based on similar concepts of building an accurate learner profile from interaction with the PLE and the social media being employed. The intention of these sensors was ultimately to provide accurate recommendations reflected through different output devices within the PEN. In this respect the objectives identified were to:

1. Employ wearable devices as input sensors to collect learner information to contribute to the profile generation process.

2. Make use of the learner's mobile phone as an input device when extracting information from the WWW and from the surrounding environment.

3. Make use of a tablet or the same mobile phone as output devices to notify the user about the surroundings and to be used as the main communication media.

The information about the learner in this case was also extracted from social networks and to these ends Facebook profiles tend to be much more indicative about their users given the type of information people share. Facebook's Graph API was therefore used to obtain the desired information which information varies from personal information, such as demographics, to particular likes such as interests, artistic groups, and so on. The information is supplemented with information from the user's social media feed giving it a much needed contextual grounding. Similar to the PLE much of the information was implicitly extracted requiring only a minimal amount of explicit data input from the user. The user's hundred most recent likes and twenty-five most recent posts to the media profile were taken into consideration and were later employed to create the required personalisation and profile generation. This amount of data is ideal since it finds a balance between having just about the right amount of data to be able to perform user profiling without overloading the application with information to be sent to the server, and eventually read back, making the application process too slow.

The gathered data was processed using a number of techniques to extract the raw information required. Analysis included tokenisation, stop-word removal, ontology assimilation through WordNet, and finally synonyms generation. The user profile was then generated through the use of Term Frequency-Inverse Document Frequency (TD-IDF), a basic statistical technique that computes the importance of a particular word within a document in respect to a collection or corpus of documents. The most important words were then in turn used to base any assumptions for building the user profile. This reasoning stems from the thought that if a learner talks or searches about some specific item, then one can deduct that the learner is interested in this item. Consequently these most commonly occurring words are considered to be indicative of the user's interests, and for the purpose of this prototype the two hundred most commonly occurring words were taken into consideration giving a sufficiently clear dataset on which to perform the remaining tasks. The user-profiling technique adoptedemployed a hybrid approach between Weighted Key-word representation and the Semantic representation, but eventually the categorisation of the learner's interest was clusteredinto a specific groups in order to improve uniformity. There are twelve identified categories which are: "photography", "shopping", "history", "military", "food", "religion", "art", "technology", "science", "music", "sport" and "nature". The profiling process was split up into three phases in order to ensure utmost veracity when the final results were achieved. The first stage involved comparing a set of words, deemed 
to be the most frequently used words by the user after analysis of the gathered data to the groups that correspond best to the interests categories. Thus if the list of most commonly occurring words contains some word that is found in the list of identified interests, then that particular interest category is marked as relevant. Although this is one form of classifying the user, it was deemed too trivial and too risky when considering the result accuracy. As a second measure of profiling the word ontology results were introduced by which the system compares the synonyms of the most frequently used words to the stereotype categories. To further complement this, in the final stage of categorization, the system also looked at the synonyms of the landmark types and performed one final check in order to categorize the user into the most representative categories based on his interests. That ensures that if the list of most commonly occurring words does not contain the exact name of an interest field, then more checks are carried out to increase the chances of obtaining a hit. At the end of this cycle the result would be a user profile consisting of the interest fields that are deemed to be of interest to the user.

The intelligent wearables were used as both input and output media to help integrate learners with the personal learning environment and also together. Recommendations generated through the identification of interest fields were communicated through a wrist band and hyperlinked to educational content within the correct context. Tests using iWatch ${ }^{3}$ and Fitbit ${ }^{4}$ have shown that seamless learner notification together with customised content according to the specific interest adds value and enhances student understanding especially if depicted within an ideal context [69]. Input wearable devices are more limited but the use of the wristband served also to pair up learners with common interests. The different user profiles were coded onto the wristband from the backend server and once one wristband identifies the vicinity of another matching wristband a vibrating notification alerts both learners. Such methodologies were already found to be effective [70] and their use highly recommended at all level of education. In this particular context their combination with other technologies within a higher education scenario they tend to be more effective and inspirational.

\subsection{Cognisant Environment}

The integration of the previous two technologies together was augmented once the environment or the ambient around the learners was aware and reactive. Beacons were positioned with the vicinity of four labs to identify specific learners and direct them towards the appropriate lab. Each lab had a specific setup associated with part of the syllabus being covered and the learners were asked to interact with materials and educational resources associated to their interests. The four labs were setup with the following themes: Robotics, Vision and Image Processing, Mobile Technologies, and Games. Communication to the wristbands and to the mobile devices enabled the learners to arrive at the correct lab which their personal learning environment directed them to in synchronisation with the sequence of the academic material and with their identified interest. This simulated ambient intelligent setup served as a prototype setting to combine all the three technologies together to embody all the required elements of a complete educational institution.

\footnotetext{
${ }^{3}$ http://www.apple.com/watch/

${ }^{4}$ https://www.fitbit.com/eu
} 


\section{TESTING}

Each individual component was tested in isolation with the latter ambient intelligent technology making use of the former two. The intelligent personal learning environment was tested with a hundred and twenty higher education participants with positive results [71] as it was compared to the traditional face-to-face medium and the classical static e-learning environment. The study showed that by combining three techniques, crowdsourcing, learner profiling and personalisation, into a functional system it was possible to enhance effectivenessby delivering a tailored environment that adapts to the learner. It also showed that it is possible to make use of social networks to crowdsource additional information to supplement the available academic content.

The intelligent wearables were also tested with a smaller group of forty-five university students who were first asked to explicitly mark which of the interest fields they thought best described their interests, and later asked to make use of the system together with purposely purchased wristbands. All the students owned a smartphone and tested the PLE over their mobile device while networked with the wristband and the backend application. The system extracted the participants' social media profiles and generated a learner profile accordingly, and eventually compared to the interest fields marked by the learners. The results showed an average precision rate of $81 \%$ when the system classified the learner into one correct category and return relevant suggestions. The recall values returned by the system ranged from 60 to $100 \%$ when relevant interest field were found in the learner profile. Students were also asked about the general deployment of the wearable devices and the use of their smartphone as part of their academic experience. The feedback was astounding as the use of such devices tended to blend much more with their everyday interaction outside academia and thereby resulted in a much more natural interaction rather than a forced one.

Finally the use of simulated ambient intelligence was evaluated as the students were asked to comment about the use of the labs.

\section{FUTURE WORK}

More work could be done to improve the performance of the system with respect to its personalisation capabilities, more specifically, to improve on the Ontology-based approach adopted in this study. The first issue that should be tackled is the cold-start problem that the system might encounter when working on some profiles. This problem could be tackled by looking at alternative sources through which it could acquire data for personalisation, which may be other social media platforms or through mild forms of explicit data gathering. Also, the use of a hybrid approach to personalisation would perhaps be ideal. Boosting the personalisation capabilities of the system could also be achieved through obtaining more information about the user from other sources. There is a reluctance to move towards explicit data gathering but the need for better input data is clear. This can be achieved from other sources such as a user's browser history. Future research should focus on how e-learning turns the tables on the learners in relation to participation, behaviour and required effort. What methodology should be adopted to effectively introduce the learners to the e-learning platform? Which learning theory subscribes to such a philosophical undertaking? How can resistance to change be controlled and actually reverse the learners' mind-set? How will this be implemented and eventually performed in reality?Other future research directions might include the customisability and control of the environment by the learner, especially in the tertiary level. Participants pointed out that they 
would have recommended the iPLE to their peers if they could switch it on and off at their will. This interesting concept emulates the behaviour of online learners who are very focused when they have a specific objective to reach, but willing and open to related suggestions and recommendations when they are following a course, like a MOOC, out of interest and without any assessment repercussions or time restrictions. Another research direction can potentially be within the crowdsourcing domain where additional sources could be automatically included and harvested to add richer and diverse content to the knowledge base of the iPLE. The current version collates information from pre-stated sources on specific interest areas. An extensible and fully automated system would be able to enhance the repertoire, or even better, refine the interests categories thereby optimising further more the personalisation process of the e-learning environment.

\section{CONCLUSiOnS}

This paper has been a tiny step towards the right direction. An attempt into how to optimise elearning through a personal education network. The research that pursued following this goal characterised the design, methodologies adopted and the actual development of the prototypes presented and evaluated. The evaluation concluded that the goals and objectives of the research have been successfully met or exceeded. This research study and this paper do not only recapitulate all the hard work performed over the last four years, and nor do they characterise the end of an exhilarating journey, but merely demarcate the beginning of a promising way forward as new research avenues have been uncovered which potentially could characterise the future of online education and intelligent e-learning platforms and personal educational networks. This is all very promising and encouraging because the work presented helps to improve and enhance people's interaction and attitude towards e-learning and online education in general.

\section{REFERENCES}

[1] M. McAleese et al., "Improving the quality of teaching and learning in Europe's higher education institutions," High Level Group on the Modernisation of Higher Education, Report to the European Commission, Luxembourg, ISBN 978-92-79-30360-9, 2013.

[2] M Yorke and B. Longden, Retention and Student Success in Higher Education. Maidenhead, UK: Open University Pres, 2004.

[3] L. P. Macfadyen and S. Dawson, "Numbers Are Not Enough. Why e-Learning Analytics Failed to Inform an Institutional Strategic Plan," Educational Technology \& Society, vol. 15, no. 3, pp. 149163, 2012.

[4] B. Park-Woolf, Building Intelligent Interactive Tutors : Student-Centered Strategies for Revolutionizing E-learning. Burlington, MA, USA: Morgan Kaufmann, 2010.

[5] N. Sclater, "Web 2.0, Personal Learning Environments, and the future of Learning Management Systems," Colorado, 2008.

[6] S. Leone, Characterisation of a Personal Learning Environment as a Lifelong Learning Tool. Ancona: Springer, 2013.

[7] S. Gooren-Sieber and A. Henrich, "Systems for Personalised Learning: Personal Learning Environment vs. E-Portfolio?," in Hybrid Learning. Germany: Springer, 2012, pp. 294-305.

[8] D. Morrison. (2013, January) Online Learning Insights. [Online]. https://onlinelearninginsights.wordpress.com/2013/01/30/why-students-need-personal-learningportfolios-more-than-we-do/

[9] L.P. Cox, "Truth in Crowdsourcing," IEEE Journal on Security and Privacy, pp. 74-76, 2011.

[10] A.D. Ramakrishnan and A.Y. Halevy, "Crowdsourcing systems on the World-Wide Web," Communications of the ACM, vol. 54, no. 4, pp. 86-95, 2011. 
International Journal of Education (IJE) Vol.4, No.4, December 2016

[11] M. Bernstein et al., "Soylent: A word processor with a crowd inside," in UIST, 2010.

[12] M. Bernstein, J. Brandt, R. Miller, and D. Karger, "Crowds in two seconds: Enabling realtime crowdpowered interfaces," in UIST, 2011.

[13] V.A. Fitt, "Crowdsourcing the News: News Organizaion Liability for iReporters," William Mitchell Law Review, pp. 1839-1867, 2011.

[14] D.P. Casal, "Crowdsourcing the Corpus: Using Collective Intelligence as a Method for Composition," Leonardo Music Journal, pp. 25-28, 2011.

[15] T.C. Norman, C. Bountra, A.M. Edwards, K.R. Yamamoto, and S.H. Friend, "Leveraging Crowdsourcing to Facilitate the Discovery of New Medicines," Science Translational Medicine, 2011.

[16] Bommert, "Collaborative innovation in the public sector," International Public Management Review, pp. 15-33, 2010.

[17] P. Belleamme, T. Lambert, and A. Schwienbacher, "Crowdfunding: Tapping the Right Crowd," in The International Conference of the French Finance Association, 2010.

[18] I. Literat, "The Work of Art in the Age of Mediated Participation: Crowdsourced Art and Collective Creativity ," International Journal of Communication, pp. 2962-2984, 2012.

[19] J. Costa, C. Silva, M. Antunes, and B. Ribeiro, "On using crowdsourcing and active learning to improve classification performance," in 11th International Conference on Intelligent Systems Design and Applications (ISDA), Cordoba, 2011, pp. 469 - 474.

[20] D.S. Weld et al., "Personalised Online Education - A Crowdsourcing Challenge," in AAAI Workshops at Twenty-Sixth AAAI Conference on AI, 2012, pp. 159-163.

[21] E. Bonabeau, "Decisions 2.0: the power of collective intelligence," MIT Sloan Management Review, pp. 45-52, 2009.

[22] D. Cosley, D. Frankowski, L. Terveen, and J. Riedl, "Suggestbot: Using intelligent task routing to help people find work in wikipedia," in Conference on Intelligent User Interfaces, 2007.

[23] R. Junco, G.. Heiberger, and E. Loken, "The effect of Twitter on college student engagement and grades," Journal of Computer Assisted Learning, 2010.

[24] C. Rutherford, "Using Online Social Media to Support Preservice Student Engagement," MERLOT Journal of Online Learning and Teaching, vol. 6, no. 4, pp. 703-711, 2010.

[25] G. Lorenzo and J. Ittelson, "An Overview of E-Portfolios," Educase Learning Initiative, 2005.

[26] A.L. Daunert and L. Price, "E-Portfolio: A Practical Tool for Self-Directed, Refl ective, and Collaborative Professional Learning," in Discourses on Professional Learning: On the Boundary Between Learning and Working. Dordrecht: Springer Science+Business Media , 2014, pp. 231-251.

[27] H. Yongqiang and Y. Jinwu, "Study on the Evaluation System of E-Learning Based on E-Learning Portfolio," in ICCIC 2011, Heidelberg, 2011, pp. 420-426.

[28] M.D. D'Alessandro, "Connecting your radiology learning to your clinical practice: using personal learning environments, learning portfolios and communities of practice," Pediatr Radiol, vol. 41, pp. 245-246, 2011.

[29] Z. Guo and J. Greer, "Electronic Portfolios as a Means for Initializing Learner Models for Adaptive Tutorials," in EC-TEL 2006, Heidelberg, 2006, pp. 482 - 487.

[30] S. Schiaffino and A. Amandi, "Intelligent User Profiling," in Artificial Intelligence, LNAI 5640.: IFIP, 2009, pp. $193-216$.

[31] J., Ortega, F., Hernando, A., Bernal, J. Bobadilla, "A collaborative filtering approach to mitigate the new user cold start problem," Knowledge-Based Systems, pp. 225-238, 2012.

[32] I. Zukerman and D. Albrecht, "Predictive Statistical Models for User Modeling," User Modeling and User-Adapted Interaction, vol. 11, no. 1-2, pp. 5-18, 2001.

[33] P. Brusilovsky, O. Stock, and C. Strapparava, Adaptive Hypermedia and Adaptive Web-Based Systems. Trento: Springer, 2000.

[34] M. Degemmis, P. Lops, G. Semeraro, and F. Abbattista, "Extraction of User Profiles by Discovering Preferences through Machine Learning," in Intelligent Information Processing and Web Mining. Zakopane, Poland: Springer, 2003, pp. 69-78.

[35] S. Gauch, M. Speretta, A. Chandramouli, and A. Micarelli, "User Profiles for Personalized Information Access," in The Adaptive Web. Heidelberg: Springer, 2007, pp. 54-89. 
[36] A. Cawsey, F. Grasso, and C. Paris, "Adaptive Information for Consumers of Healthcare," in The Adaptive Web. Heidelberg: Springer, 2007.

[37] A. Goy, L. Andrissono, and G. Petrone, "Personalization in E-Commerce Applications," in The Adaptive Web. Hesidelberg: Springer, 2007, pp. 485-520.

[38] A. Krüger, J. Baus, D. Heckmann, M. Kruppa, and R. Wasinger, "Adaptive Mobile Guides," in The Adaptive Web. Heidelberg: Springer, 2007, pp. 521-549.

[39] P. Brusilovsky and E. Millán, "User Models for Adaptive Hypermedia and Adaptive Educational Systems," in The Adaptive Web. Heidelberg: Springer, 2007, pp. 3-53.

[40] M. Vargas-Vera and M. Lytras, "Exploiting semantic web and ontologies for personalised learning services: towards semantic web-enabled learning portals for real learning experiences," International Journal of Knowledge and Learning, vol. 4, no. 1, pp. 1-17, 2008.

[41] D. Dagger, V. Wade, and O.T. Conlan, ""anytime, anywhere" learning: The role and realization of dynamic terminal personalization in adaptive learning," in Ed-Media 2003: World Conference on Educational Multimedia, Hypermedia and Telecommunications, 2003.

[42] N. Manouselis and D. Sampson, "Dynamic Knowledge Route Selection for Personalised Learning Environments using Multiple Criteria," in Intelligence and Technology in Education Applications Workshop - ITEA2002, Innsbruck, 2002.

[43] M. Van Harmelen, "Personal Learning Environments," in Sixth IEEE International Conference on Advanced Learning Technologies, Washington, 2006, pp. 815-816.

[44] G. Siemens. (2012) Connectivism. [Online]. www.connectivism.ca

[45] D. Gurzick and K. White, "Online Personal Networks of Knowledge Workers in Computer-Supported Collaborative Learning," in Computer-Supported Collaborative Learning at the Workplace. New York: Springer, 2013, pp. 225-239.

[46] Knewton. (2016, January) Knewton in the News. [Online]. https://www.knewton.com/resources/press/pearson-and-knewton-team-up-to-personalize-matheducation/

[47] CogBooks. (2015, September) Using adaptive learning tools - An educator's perspective. [Online]. https://www.cogbooks.com/2015/09/15/using-adaptive-learning-tools-an-educators-perspective/

[48] O. Lawlor. (2015, July) Metacog releases open ended rubric based machine scoring service. [Online]. http://www.metacog.com/blog/files/category-assessment.html

[49] CogBooks. (2016, February) mprove Student Success and Retention with Adaptive Courseware. [Online].https://www.cogbooks.com/2016/02/04/improve-student-success-and-retention-withadaptive-courseware/

[50] D.M. Reddy, "U-Pace," University of Wisconsin-Milwaukee, 2014.

[51] MIT. (2016, January) Free online courses from MIT. [Online]. https://www.edx.org/school/mitx [52]IMS, "Learning Measurement for Analytics Whitepaper," IMS Global Learning Consortium, Inc., 2013.

[53] Educause. (2016, February) Personalized Learning. [Online]. https://library.educause.edu/topics/teaching-and-learning/personalized-learning

[54] S. Lonn, A. Nixon, G. Morgan, C. VanDenBlink, and E. Dahlstrom, "Moving the Red Queen Forward: Maturing Learning Analytics Practices," in Educause15, 2015.

[55] J.P. Redlich, W. Müller, H. Plötz, and M. Stigge. (2005) Secure Personal Area Networks (SPAN). [Online]. https://sar.informatik.hu-berlin.de/research/projects/2005-SPAN/span.htm

[56] M. Montebello, "Enahncing e-learning through the merging of e-portfolios, social networks and artificial intelligence," in 10th International Technology, Education and Development Conference, Valencia, 2016.

[57] L.A. Tomei, "Learning Theories and Pedagogy: Teaching the Traditional Learner," in Designing Instruction for the Traditional, Adult, and Distance Learner: A New Engine for Technology-Based Teaching. USA: IGI Global, 2010, pp. 1-14.

[58] J. McKenzie, "Pedagogy does matter," The Educational Technology Journal, vol. 13, no. 1, 2003.

[59] G. Siemens. (2004) elearnspace. [Online]. http://www.ingedewaard.net/papers/connectivism/2005_siemens_ALearningTheoryForTheDigitalAg e.pdf 
[60] G.V. Ureña and J.R. Valenzuela-González, "Online social network contacts as information repositories," International Journal of Educational Technology in Higher Education, vol. 8, no. 1, pp. 142-155, 2011.

[61] W. Ng, "Theories Underpinning Learning with Digital Technologies," in New Digital Technology in Education. Switzerland: Springer International Publishing, 2015, pp. 73-94.

[62] T. Mayes and S. de Freitas, "Technology_Enhanced learning: The role of theory," in Rethinking pedagogy for a digital age: Designing for 21 st century learning. New York: Routledge, 2013, pp. 1730 .

[63] G. Siemens. (2004, Dec.) elearnspace. [Online]. http://www.elearnspace.org/Articles/connectivism.htm

[64] L. Vygotsky, Mind in society: The development of higher psychological processes. Cambridge: Harvard University Press, 1978.

[65] A. Loureiro and T. Bettencourt, "Immersive Environments - A Connectivist Approach," in WSKS 2010, Part I, CCIS 111. Berliin: Springer-Verlag, 2010, pp. 202-214.

[66] R. Robson, "The Changing Nature of E-Learning Content," in Reshaping Learning - Frontiers of Learning Technology in a Global Context. Berlin: Springer-Verlag, 2013, pp. 177-196.

[67] B. Duke, G. Harper, and M. Johnston, "Connectivism as a Digital Age Learning Theory," The International HETL Review, 2013.

[68] N. M. Hung, "Using Ideas from Connectivism for Designing New Learning Models in Vietnam," International Journal of Information and Education Technology, vol. 4, no. 1, pp. 76-82, 2014.

[69] T. Naga-Swathi and S. Langa, "Wearable technology a new paradigm in Educational Universities," International Journal on Computer Science and Engineering (IJCSE), vol. 7, no. 4, pp. 48-52, 2015.

[70] V. Prabhu, T. Olsson, and et. al., "How Can Wearables Support CoLocated Social Interaction?," in NordiCHI'14, Helsinki, Finland., 2014, pp. 26-30.

[71] M. Montebello, "Enhancing e-learning through the merging of e-portfolios, social networks and artificial intelligence," in. 10th International Technology, Education and Development Conference , Valencia, Spain, 2016. 